\title{
Time frequency features of rotor systems with slowly varying mass
}

\author{
Tao Yu ${ }^{\mathrm{a}, *}$ and Qingkai $\operatorname{Han}^{\mathrm{b}}$ \\ ${ }^{a}$ School of Electromechanical Automobile Engineering, Yantai University, Yantai, 264005, China \\ ${ }^{\mathrm{b}}$ School of Mechanical Engineering and Automation, Northeastern University, Shenyang, 110004, China
}

Received 11 February 2010

Revised 24 May 2010

\begin{abstract}
With the analytic method and numerical method respectively, the asymptotic solutions and finite element model of rotor system with single slowly varying mass is obtained to investigate the time frequency features of such rotor system; furthermore, with given model of slowly varying mass, the rotor system with dual slowly varying mass is studied. For the first order approximate solution is used, there exists difference between the results with analytic method and numerical method. On the base of common characteristics of rotor system with dual slowly varying mass, the general rules and formula describing the frequency distribution of rotor system with multiple slowly varying mass are proposed.
\end{abstract}

Keywords: Rotor system, slowly varying mass, FEM, analytic method, reassigned Morlet scalograms

\section{Introduction}

In detaching machine, weaving machine and hoisting device, etc, the mass of the rotating part changes continuously. For example, in weaving machine, the mass of spindle changed slowly with the amount of thread winding on it. Theoretically, the rotor system, whose system parameters related with mass, i.e. rotor mass, changed slowly with time, is called rotor system with slowly varying mass. Obviously, the continuous change of system mass will result in different dynamics response compared with the constant mass system. So far, studies on the rotor system with slowly varying mass are not enough [1]. In 50s of 19th century, the nonlinear equations with slow varying parameters was studied by Mitropolsky [2], he advanced an asymptotic method of system with slow-changing parameters. Based on the technique of freezing slowly changing parameters, Shahruz derived an explicit approximate solution of a linear slowly varying system [3]. In recent years, Cveticanin studied the chaos characteristics and normal mode of vibration in rotor system with slowly varying mass $[4,5]$. Bangchun Wen promote the studies on rotor system with slowly varying parameters, especially on the aspect of slowly varying stiffness [6]. Yinong Li studied the dynamic characteristics of slowly changing process of a vibration system, and gave relationship between the amplitude and the frequency of the system [7]. In addition, the studies focused on strong nonlinear oscillators with slowly varying parameters were carried out with multiple scales and KBM methods [8-10].

In the paper, the nonlinear characteristics of rotor system with slowly varying mass will be studied, and some interesting phenomena and important results will be given.

\footnotetext{
${ }^{*}$ Corresponding author. E-mail: yt_126@126.com.
} 


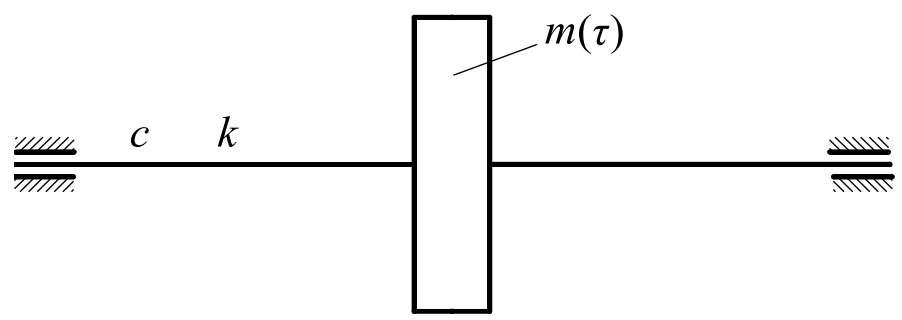

Fig. 1. Simplified-lumped-mass rotor system with slowly varying mass.

\section{Analytic method for a simplified-lumped-mass rotor system with slowly varying mass}

\subsection{Dynamic equations of slowly varying mass rotor system}

A simplified Jeffcott rotor system, as shown in Fig. 1, its mass is slowly varying with time. The dynamic equations can be expressed as:

$$
\left\{\begin{array}{l}
\frac{\mathrm{d}}{\mathrm{d} t}\left[m(\tau) \frac{\mathrm{d} x}{\mathrm{~d} t}\right]+c \frac{\mathrm{d} x}{\mathrm{~d} t}+k x=m(\tau) e \omega^{2} \cos (\omega t) \\
\frac{\mathrm{d}}{\mathrm{d} t}\left[m(\tau) \frac{\mathrm{d} y}{\mathrm{~d} t}\right]+c \frac{\mathrm{d} y}{\mathrm{~d} t}+k y=m(\tau) e \omega^{2} \cos (\omega t)-m(\tau) g
\end{array}\right.
$$

where $m(\tau)$ is the slowly varying mass of rotor system, $c$ is the damping coefficient, $k$ is the linear stiffness of rotor shaft, $g$ is the gravity.

Since slowing varying time $\tau=\varepsilon t$, we have $\frac{\mathrm{d}}{\mathrm{d} t}=\frac{\mathrm{d}}{\mathrm{d} \tau} \frac{\mathrm{d} \tau}{\mathrm{d} t}=\varepsilon \frac{\mathrm{d}}{\mathrm{d} \tau}$, and the Eq. (1) can be reformulated as:

$$
\left\{\begin{array}{l}
\frac{\mathrm{d}^{2} x}{\mathrm{~d} t}+\omega_{0}^{2}(\tau) x=-\frac{c+\varepsilon m^{\prime}(\tau)}{m(\tau)} \frac{\mathrm{d} x}{\mathrm{~d} t}+e \omega^{2} \cos (\omega t) \\
\frac{\mathrm{d}^{2} y}{\mathrm{~d} t}+\omega_{0}^{2}(\tau) y=-\frac{c+\varepsilon m^{\prime}(\tau)}{m(\tau)} \frac{\mathrm{d} y}{\mathrm{~d} t}+e \omega^{2} \cos (\omega t)-g
\end{array}\right.
$$

where $\omega_{0}^{2}(\tau)=k / m(\tau), m^{\prime}(\tau)=\mathrm{d} m(\tau) / \mathrm{d} \tau$.

Compared with the rotor system with constant mass, the system with slowing varying mass has an extra item of damping, which is the function of slowly varying mass. If the mass change rate is positive, the slowly varying damping coefficient is positive; otherwise it becomes negative and can cause self-excited vibration. In addition, the natural frequency of rotor system with slowly varying mass is varying slowly too, which can incur the slow varying of vibration amplitude and phase angle. Under the circumstance of small damping force and exciting force, let $c / m(\tau)=2 \varepsilon \xi \omega_{0}(\tau), e=\varepsilon r$, Eq. (1) can be rewritten as [11-13]:

$$
\left\{\begin{array}{l}
\frac{\mathrm{d}^{2} x}{\mathrm{~d} t^{2}}+\omega_{0}^{2}(\tau) x=-\varepsilon\left[2 \xi \omega_{0}(\tau)+m^{\prime}(\tau) / m(\tau)\right] \frac{\mathrm{d} x}{\mathrm{~d} t}+\varepsilon r \omega^{2} \cos (\omega t) \\
\frac{\mathrm{d}^{2} x}{\mathrm{~d} t^{2}}+\omega_{0}^{2}(\tau) y=-\varepsilon\left[2 \xi \omega_{0}(\tau)+m^{\prime}(\tau) / m(\tau)\right] \frac{\mathrm{d} y}{\mathrm{~d} t}+\varepsilon r \omega^{2} \sin (\omega t)-g
\end{array}\right.
$$

Since the two equations of Eq. (3) are irrelative, the functions can be solved separately. Take the horizontal equation as example, it can be rewritten as:

$$
\begin{aligned}
\frac{\mathrm{d}^{2} x}{\mathrm{~d} t^{2}}+\omega_{0}^{2}(\tau) x & =-\varepsilon\left[2 \xi \omega_{0}(\tau)+m^{\prime}(\tau) / m(\tau)\right] \frac{\mathrm{d} x}{\mathrm{~d} t}+\varepsilon r \omega^{2} \cos (\omega t) \\
& =\varepsilon\left\{-\left[2 \xi \omega_{0}(\tau)+m^{\prime}(\tau) / m(\tau)\right] \frac{\mathrm{d} x}{\mathrm{~d} t}+r \omega^{2} \cos (\omega t)\right\} \\
& =\varepsilon f\left(\tau, t, \frac{\mathrm{d} x}{\mathrm{~d} t}\right)
\end{aligned}
$$

where, $f\left(\tau, t, \frac{\mathrm{d} x}{\mathrm{~d} t}\right)=-\left[2 \xi \omega_{0}(\tau)+m^{\prime}(\tau) / m(\tau)\right] \frac{\mathrm{d} x}{\mathrm{~d} t}+r \omega^{2} \cos (\omega t)$ is a nonlinear item. Expand $f\left(\tau, t, \frac{\mathrm{d} x}{\mathrm{~d} t}\right)$ as Fourier series, we have

$$
f\left(\tau, t, \frac{\mathrm{d} x}{\mathrm{~d} t}\right)=\sum_{n=-N}^{N} f_{n}\left(\tau, t, \frac{\mathrm{d} x}{\mathrm{~d} t}\right) \cdot e^{i n \omega t}
$$


Assume the solution of the Eq. (5) has the form like

$$
x=a \cos (\omega t+\psi)+\varepsilon u_{1}(\tau, a, t, \psi)+\varepsilon^{2} u_{2}(\tau, a, t, \psi)+\cdots
$$

Where, both $a$ and $\psi$ are the function of time $\mathrm{t} ; u_{1}(\tau, a, t, \psi), u_{2}(\tau, a, t, \psi), \ldots$ are the functions with respect to $(\omega t+\psi)$.

Assume the first order approximate solution is:

$$
\begin{aligned}
& x=a \cos (\omega t+\psi) \\
& \left\{\begin{array}{l}
\frac{\mathrm{d} a}{\mathrm{~d} t}=\left[\varepsilon \delta_{1}(\tau, a, \psi)+\varepsilon^{2} \delta_{2}(\tau, a, \psi)\right] a+\cdots \\
\frac{\mathrm{d} \psi}{\mathrm{d} t}=\omega_{0}(\tau)-\omega+\varepsilon \omega_{1}(\tau, a, \psi)+\varepsilon^{2} \omega_{2}(\tau, a, \psi) \cdots
\end{array}\right. \\
& \int_{0}^{2 \pi} \frac{\mathrm{d}^{2} x}{\mathrm{~d} t^{2}}+\omega_{0}^{2}(\tau) x-\left.\varepsilon f\left(\tau, t, \frac{\mathrm{d} x}{\mathrm{~d} t}\right)\right|_{x=a \cos (\omega t+\psi)+\cdots} \cos (\omega t+\psi) \mathrm{d} \psi=0 \\
& \int_{0}^{2 \pi} \frac{\mathrm{d}^{2} x}{\mathrm{~d} t^{2}}+\omega_{0}^{2}(\tau) x-\left.\varepsilon f\left(\tau, t, \frac{\mathrm{d} x}{\mathrm{~d} t}\right)\right|_{x=a \cos (\omega t+\psi)+\cdots} \sin (\omega t+\psi) \mathrm{d} \psi=0
\end{aligned}
$$

The first order approximate solution is

$$
x=a \cos \vartheta=a \cos (\omega t+\psi)
$$

Where, $a$ and $\psi$ is the function of time $t$, and they are given as

$$
\left\{\begin{array}{l}
\frac{\mathrm{d} a}{\mathrm{~d} t}=\varepsilon \delta_{1}(\tau, a, \psi) a \\
\frac{\mathrm{d} \psi}{\mathrm{d} t}=\omega_{0}(\tau)-\omega+\varepsilon \omega_{1}(\tau, a, \psi)
\end{array}\right.
$$

The unknown function $\delta_{1}$ and $\omega_{1}$ can be obtained by solving the following equations:

$$
\begin{aligned}
& {\left[\omega_{0}(\tau)-\omega\right] a \frac{\partial \omega_{1}}{\partial \psi}+2 \omega_{0}(\tau)\left(\delta_{1} a\right) } \\
= & -\frac{1}{m(\tau)} \frac{\mathrm{d}[m(\tau) \omega(\tau)]}{\mathrm{d} \tau}-\frac{1}{2 \pi^{2} m(\tau)} \sum_{\sigma} e^{i \sigma \psi} \int_{0}^{2 \pi} \int_{0}^{2 \pi} f(\tau, a, t, \vartheta) e^{-i \sigma \psi^{\prime}} \sin (\vartheta) \omega \mathrm{d} t \mathrm{~d} \psi \\
& {\left[\omega_{0}(\tau)-\omega\right] a \frac{\partial\left(\delta_{1} a\right)}{\partial \psi}+2 a \omega_{0}(\tau) \omega_{1} } \\
= & -\frac{1}{2 \pi^{2} m(\tau)} \sum_{\sigma} e^{i \sigma \psi} \int_{0}^{2 \pi} \int_{0}^{2 \pi} f(\tau, a, t, \vartheta) e^{-i \sigma \psi^{\prime}} \cos (\vartheta) \omega \mathrm{d} t \mathrm{~d} \psi
\end{aligned}
$$

where, $\sigma$ is an integer.

$$
f(\tau, a, t, \vartheta)=f\left(\tau, \quad t, a \cos \vartheta,-a \omega_{0}(\tau) \sin \vartheta,-a \omega_{0}(\tau) \cos \vartheta\right), \quad \psi^{\prime}=\vartheta-\omega t
$$

According to the above equations, we get

$$
\begin{aligned}
\frac{\mathrm{d} a}{\mathrm{~d} t} & =-\frac{\varepsilon a}{2 m(\tau) \omega_{0}(\tau)} \frac{\mathrm{d}\left[m(\tau) \omega_{0}(\tau)\right]}{\mathrm{d} \tau}+\frac{a c}{2 m(\tau)}-\frac{\varepsilon r \omega^{2}}{\omega_{0}(\tau)+\omega} \cos \psi \\
& =-\frac{\varepsilon a}{2}\left[\frac{1}{\omega_{0}(\tau)} \frac{\mathrm{d}\left[\omega_{0}(\tau)\right]}{\mathrm{d} \tau}+\frac{1}{m(\tau)} \frac{\mathrm{d}[m(\tau)]}{\mathrm{d} \tau}\right]+a \varepsilon \xi \omega_{0}(\tau)-\frac{\varepsilon r \omega^{2}}{\omega_{0}(\tau)+\omega} \cos \psi \\
& =-\frac{\varepsilon a}{2}\left[2 \xi \omega_{0}(\tau)+\frac{\omega_{0}^{\prime}(\tau)}{\omega_{0}(\tau)}+\frac{m^{\prime}(\tau)}{m(\tau)}\right]-\frac{\varepsilon r \omega^{2}}{\omega_{0}(\tau)+\omega} \cos \psi
\end{aligned}
$$

Since we have $m(\tau)=k / \omega_{0}^{2}(\tau)$, the derivative of $m(\tau)$ can be expressed as

$$
\frac{\mathrm{d} m(\tau)}{\mathrm{d} \tau}=-\frac{2 k}{\omega_{0}^{3}(\tau)} \frac{\mathrm{d} \omega_{0}(\tau)}{\mathrm{d} \tau}
$$

The Eq. (17) can also be rewritten as 


$$
\frac{m^{\prime}(\tau)}{m(\tau)}=-2 \frac{\omega_{0}^{\prime}(\tau)}{\omega_{0}(\tau)}
$$

where, $m^{\prime}(\tau)=\frac{\mathrm{d} m(\tau)}{\mathrm{d} \tau}, \omega_{0}^{\prime}(\tau)=\frac{\mathrm{d} \omega_{0}^{\prime}(\tau)}{\mathrm{d} \tau}$.

Substitute Eq. (17) into Eq. (15), we get the solution of $a$ as

$$
\frac{\mathrm{d} a}{\mathrm{~d} t}=-\frac{\varepsilon a}{2}\left[2 \xi \omega_{0}(\tau)+\frac{m^{\prime}(\tau)}{2 m(\tau)}\right]-\frac{\varepsilon r \omega^{2}}{\omega_{0}(\tau)+\omega} \cos \psi
$$

From the Eqs (13) and (14), $\omega_{1}(\tau, a, \psi)$ can be got and then we have the solution of $\psi$ as

$$
\frac{\mathrm{d} \psi}{\mathrm{d} t}=\omega_{0}(\tau)-\omega+\frac{\varepsilon r \omega^{2}}{a\left[\omega_{0}(\tau)+\omega\right]} \sin \psi
$$

Thus, the first order approximate solution of rotor system with slowly varying mass is

$$
\left\{\begin{array}{l}
\frac{\mathrm{d} a}{\mathrm{~d} t}=-\frac{\varepsilon a}{2}\left[2 \xi \omega_{0}(\tau)+\frac{m^{\prime}(\tau)}{2 m(\tau)}\right]-\frac{\varepsilon r \omega^{2}}{\omega_{0}(\tau)+\omega} \cos \psi \\
\frac{\mathrm{d} \psi}{\mathrm{d} t}=\omega_{0}(\tau)-\omega+\frac{\varepsilon r \omega^{2}}{a\left[\omega_{0}(\tau)+\omega\right]} \sin \psi
\end{array}\right.
$$

\subsection{Wavelet analysis with given slowly varying mass model based on analytic solution}

For the detaching machine under continuously working condition, the rotor mass can be viewed as fluctuating about some certain value, and the fluctuating range and frequency are determined by the characteristics of detached material and feeding period. For convenience, the following slowly varying mass mode is examined:

$$
m(\tau)=m_{0}\left(1+\lambda \cos \omega_{m} \tau\right)
$$

The range of mass change can be easily observed as

$$
m_{0}(1-\lambda)<m<m_{0}(1+\lambda)
$$

Thus, the natural frequency of rotor system changes with the following range:

$$
\sqrt{k /\left[m_{0}(1+\lambda)\right]} / 2 \pi<f_{0}<\sqrt{k /\left[m_{0}(1-\lambda)\right]} / 2 \pi
$$

where, $k=48 E I / L^{3} ; I=\pi d^{4} / 64 ; d=2 R$ is the diameter of rotor shaft.

Assume the following parameters of rotor system, radius of rotor shaft $R=10 \mathrm{~mm}$, length of shaft $L=480 \mathrm{~mm}$, modulus $E=2.06 \mathrm{e} 11 \mathrm{~N} / \mathrm{m}^{2}$, and the stiffness of rotor $k=43888 \mathrm{~N} / \mathrm{m}$. Let $m_{0}=1 \mathrm{~kg}, \xi=0.1, e=0.0001 \mathrm{~m}$, we have the natural frequency range $27 \mathrm{~Hz}<f_{0}<47 \mathrm{~Hz}$.

Use forth order Runge-Kutta numerical method to solve Eq. (21) with $\omega_{m}=100 \mathrm{rad} / \mathrm{s}, \varepsilon=0.01$ and $\lambda=0.5$. The time-frequency response of rotor system with steady rotating speed $20 \mathrm{~Hz}, 30 \mathrm{~Hz}, 40 \mathrm{~Hz}$ and $60 \mathrm{~Hz}$ are studied.

When rotating speed is blow the range of natural frequency, as shown in Fig. 2, besides the working frequency $\omega$ the dense frequency components can be observed between the natural frequency ranges from $27 \mathrm{~Hz}$ to $47 \mathrm{~Hz}$. To examine the detail frequency components in this area, the high pass filter technology is employed. We can see from Fig. 3 that the low frequency components disappear but the dense frequency components remain obscure between $27 \mathrm{~Hz}$ from $47 \mathrm{~Hz}$. So, the reassigned Morlet scalograms method is used to analyze the filtered time-domain signals, as shown in Fig. 4. With the change of time, the frequency without working frequency component of rotor system fluctuates as characteristics of harmonic, i.e., an obvious harmonic frequency band can be observed.

When the rotor system rotates with $30 \mathrm{~Hz}$ and $40 \mathrm{~Hz}$, i.e., working between $27 \mathrm{~Hz}$ to $47 \mathrm{~Hz}$, the time-frequency responses of rotor system are shown in Fig. 5 and Fig. 6. From the two figures, it can be found that the working frequency component is submerged in the dense frequency components. So, the reassigned Morlet scalograms method is employed directly without filtering, as shown in Figs 7 and 8.

When rotor system works beyond the natural frequency range, e.g. $60 \mathrm{~Hz}$, the similar phenomenon can be observed in the Fig. 9. By using low pass filtering technique and reassigned Morlet scalograms method, the filtered time frequency diagrams and the reassigned Morlet scalograms are shown in Figs 10 and 11 respectively. 


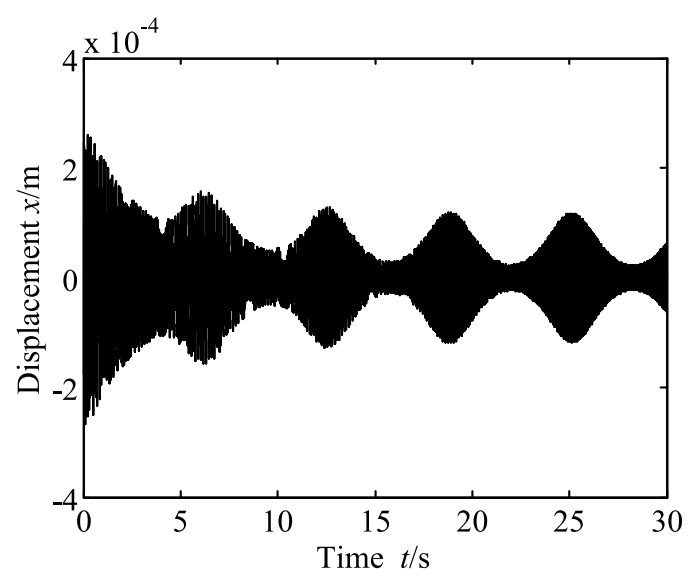

(a) Time domain signals in $x$ direction

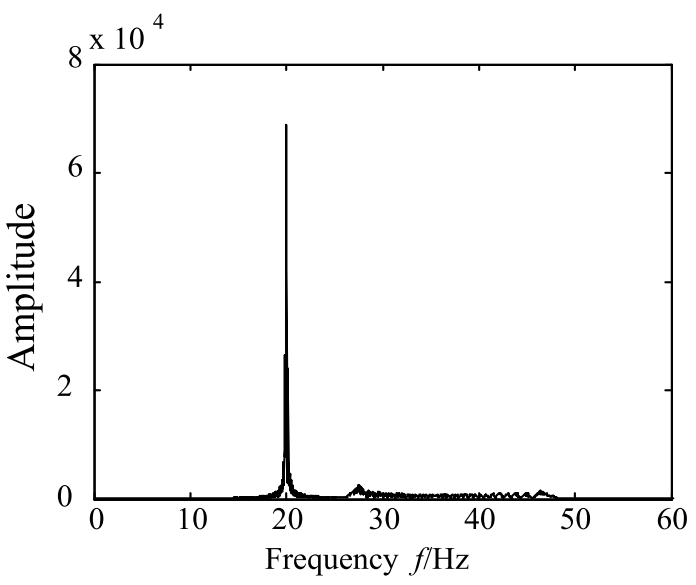

(b) Frequency spectrum

Fig. 2. Time-frequency response of rotor system with slowly varying mass $(\omega=20 \mathrm{~Hz}, \varepsilon=0.01)$.

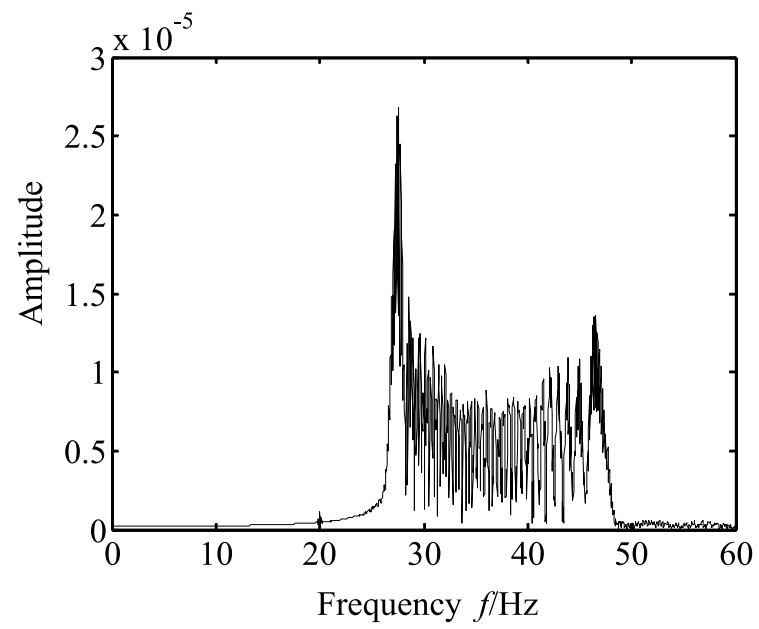

Fig. 3. Frequency spectrum of rotor system with varying mass after high-pass filtering $(\omega=20 \mathrm{~Hz}, \varepsilon=0.01)$.

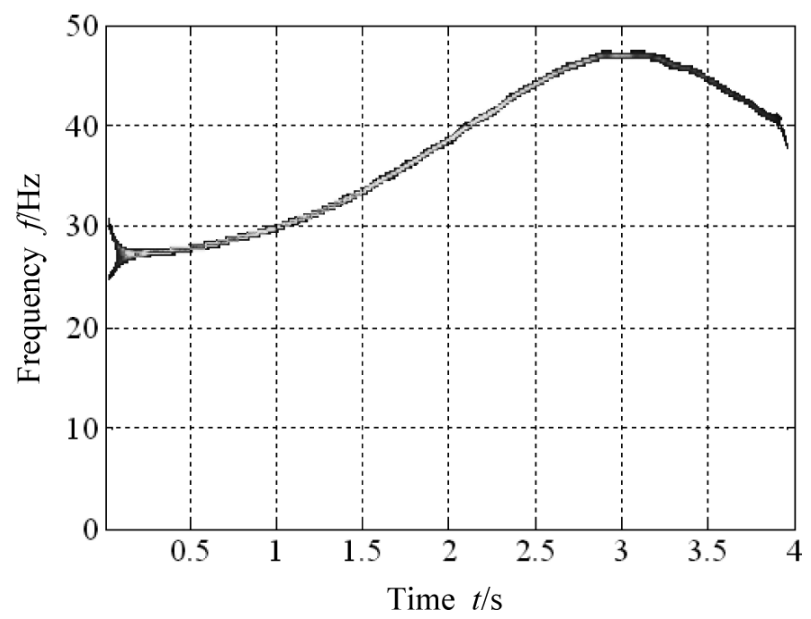

Fig. 4. The reassigned Morlet scalograms of rotor slowly system with slowly varying mass after high-pass filtering $(\omega=20 \mathrm{~Hz}, \varepsilon=0.01)$.. 


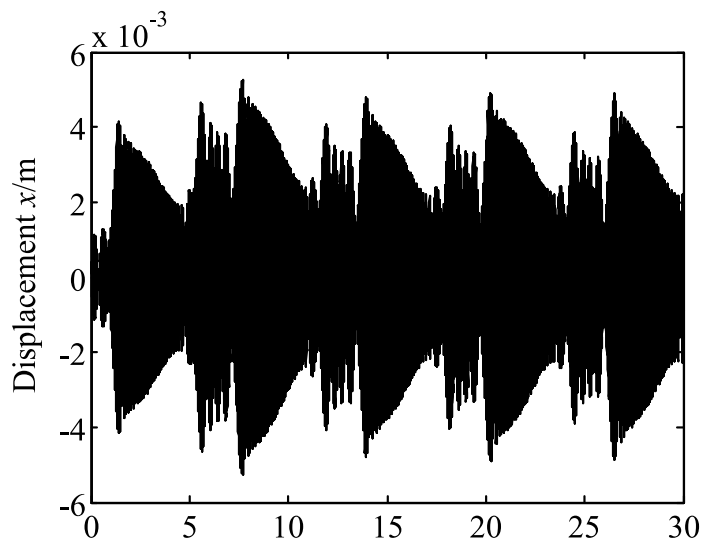

Time $t / \mathrm{s}$

(a) Time domain signals in $x$ direction

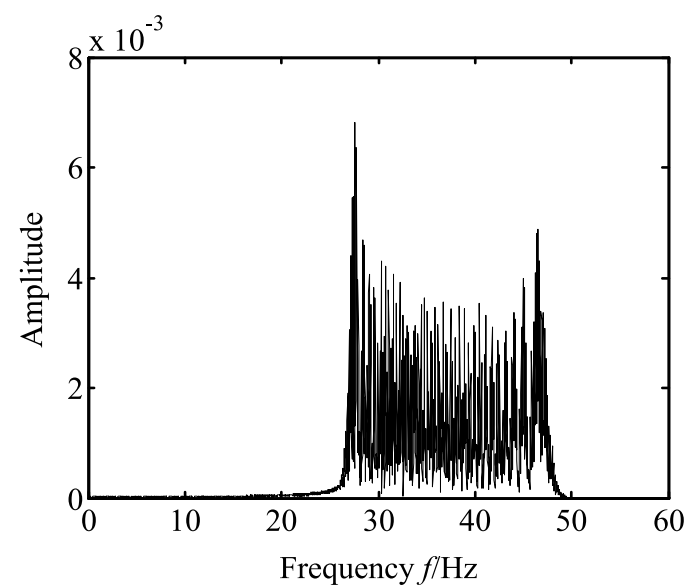

(b) Frequency spectrum

Fig. 5. Time-frequency response of rotor system with slowly varying mass after high-pass filtering $(\omega=30 \mathrm{~Hz}, \varepsilon=0.01)$.

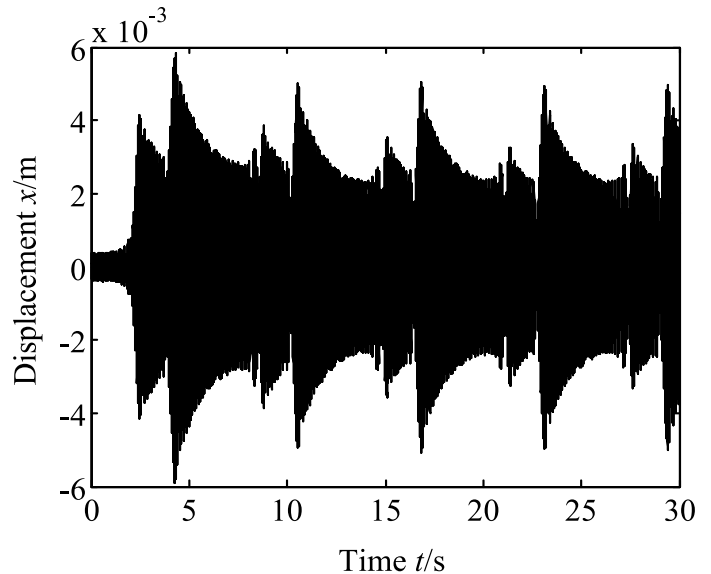

(a) Time domain signals in $x$ direction

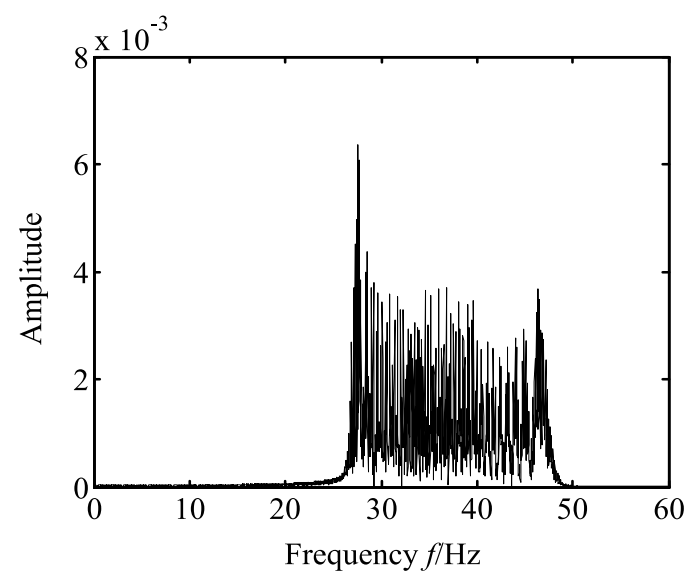

(b) Frequency spectrum

Fig. 6. Time-frequency response of rotor system with slowly varying mass after high-pass filtering $(\omega=40 \mathrm{~Hz}, \varepsilon=0.01)$.

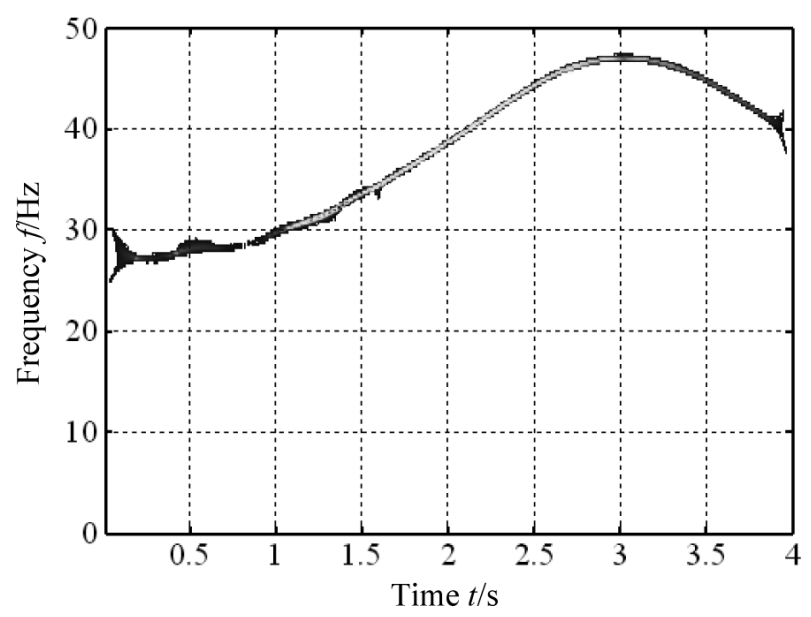

Fig. 7. The reassigned Morlet scalograms of rotor system with slowly varying mass $(\omega=30 \mathrm{~Hz}, \varepsilon=0.01)$. 


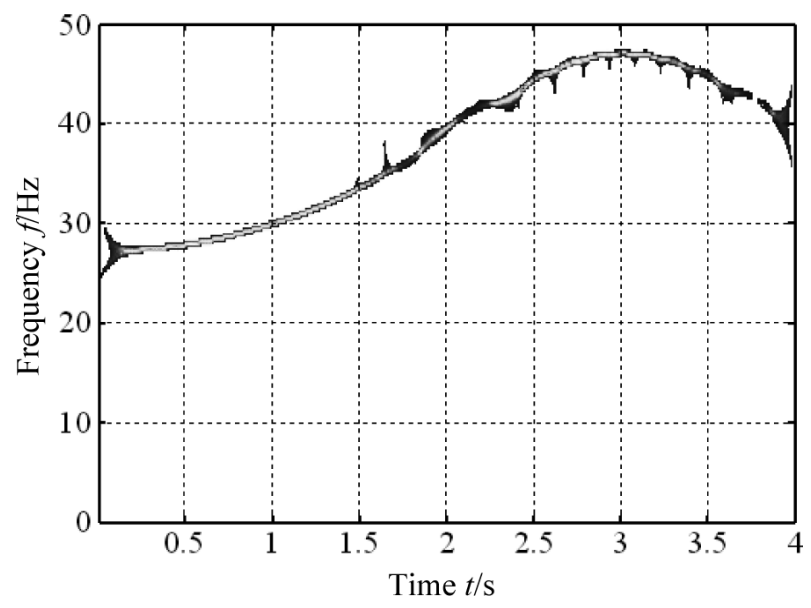

Fig. 8. The reassigned Morlet scalograms of rotor system with slowly varying mass $(\omega=40 \mathrm{~Hz}, \varepsilon=0.01)$.

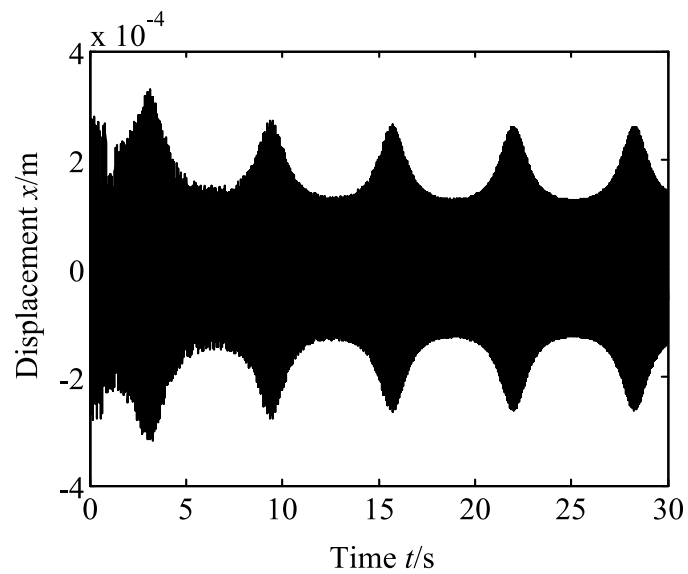

(a) Time domain signals in $x$ direction

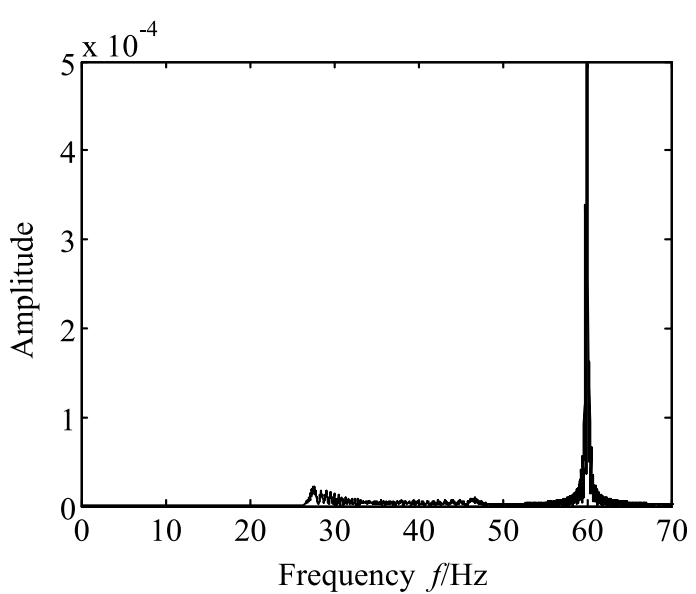

(b) Frequency spectrum

Fig. 9. Time-frequency response of rotor system with slowly varying mass after high-pass filtering $(\omega=60 \mathrm{~Hz}, \varepsilon=0.01)$.

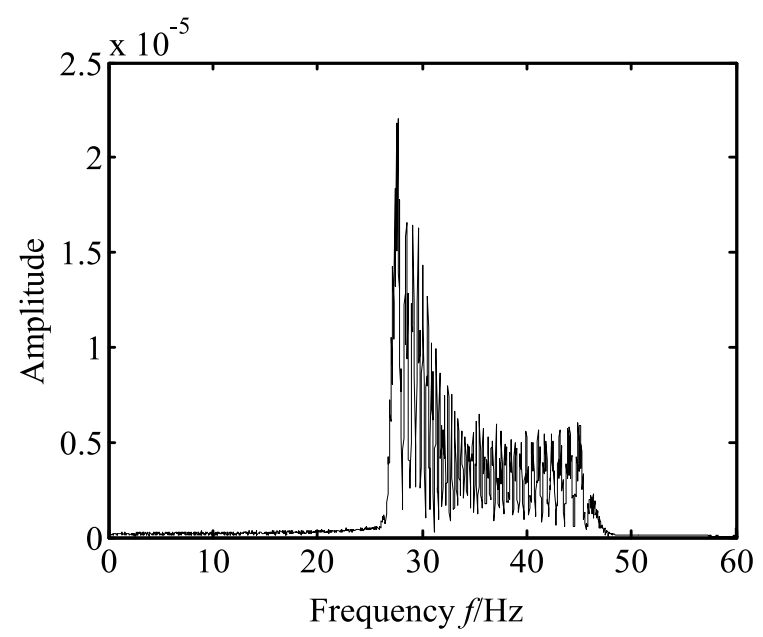

Fig. 10. Frequency spectrum of rotor system with slowly varying mass after low-pass filtering $(\omega=60 \mathrm{~Hz}, \varepsilon=0.01)$. 


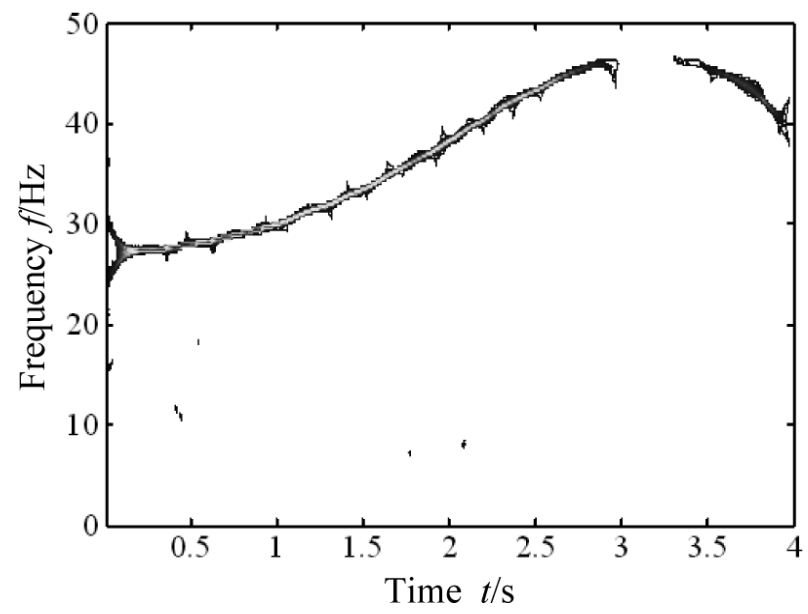

Fig. 11. The reassigned Morlet scalograms of rotor system with slowly varying mass after low-pass filtering $(\omega=60 \mathrm{~Hz}, \varepsilon=0.01)$.

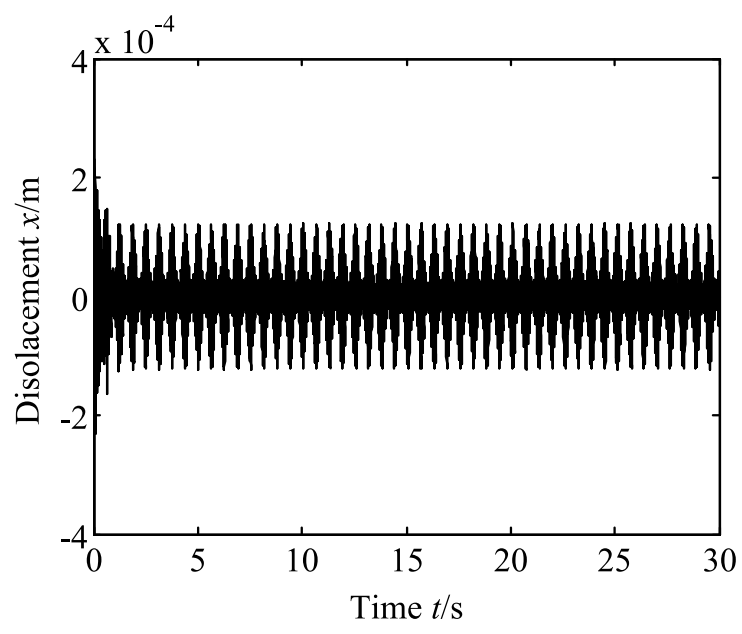

(a) Time domain signals in $x$ direction

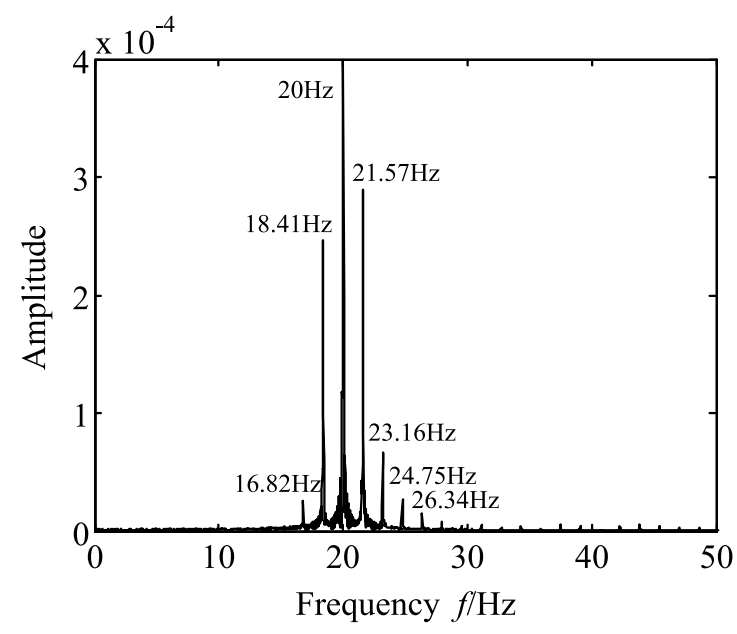

(b) Frequency spectrum

Fig. 12. Time-frequency response of rotor system with slowly varying mass $(\omega=20 \mathrm{~Hz}, \varepsilon=0.1)$.

To observe the influence of slowly varying coefficient $\varepsilon$ on the system, the case with $\omega_{m}=100 \mathrm{rad} / \mathrm{s}, \varepsilon=0.1$ and $\lambda=0.5$ is studied. Similarly, three different rotating speeds with $20 \mathrm{~Hz}, 30 \mathrm{~Hz}$ and $60 \mathrm{~Hz}$ are simulated respectively, as shown from Figs 12 to 19.

When the rotating speed is $20 \mathrm{~Hz}$, as shown in Fig. 12, the multi-frequency components can be observed around working frequency. And we can also find that the difference of these frequency components is $1.59 \mathrm{~Hz}$, which can be calculated by the following formula:

$$
\Delta f=\omega_{m} \varepsilon / 2 \pi=100 \times 0.1 / 2 / 3.14=1.59 \mathrm{~Hz}
$$

In addition, from the observation of frequency spectrum, there are the dense frequency components in high frequency area. The detailed information of those frequency components can be clearly examined by using filtering and reassigned Morlet scalogram, as shown in Figs 13 and 14. In Fig. 14, from $20 \mathrm{~Hz}$ to $50 \mathrm{~Hz}$, we find the continuous spectrum with incremental of $\Delta f$. And in Fig. 15, we find the frequency fluctuates as period of $T=2 \pi / \omega_{m} \varepsilon=1 / \Delta f$.

The cases with $30 \mathrm{~Hz}$ (between the natural frequency range) and $60 \mathrm{~Hz}$ (beyond the natural frequency range) are illustrated from Figs 15 to 19. 


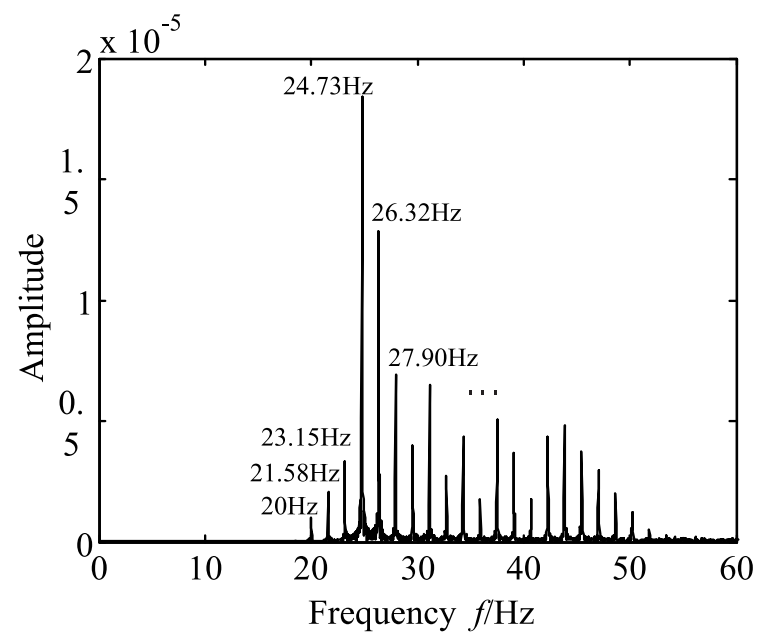

Fig. 13. Frequency spectrum of rotor system with slowly varying mass after high-pass filtering $(\omega=20 \mathrm{~Hz}, \varepsilon=0.1)$.

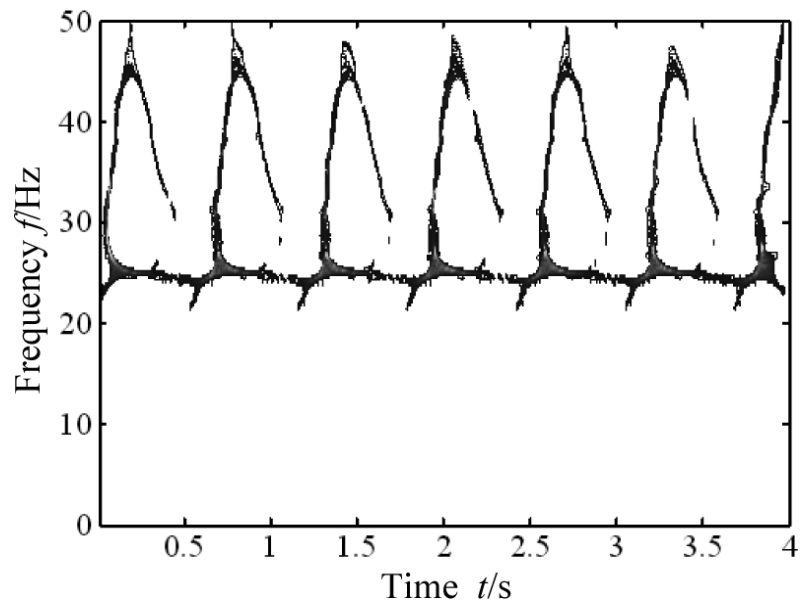

Fig. 14. The reassigned Morlet scalograms of rotor system with slowly varying mass after high-pass filtering $(\omega=20 \mathrm{~Hz}, \varepsilon=0.1)$.

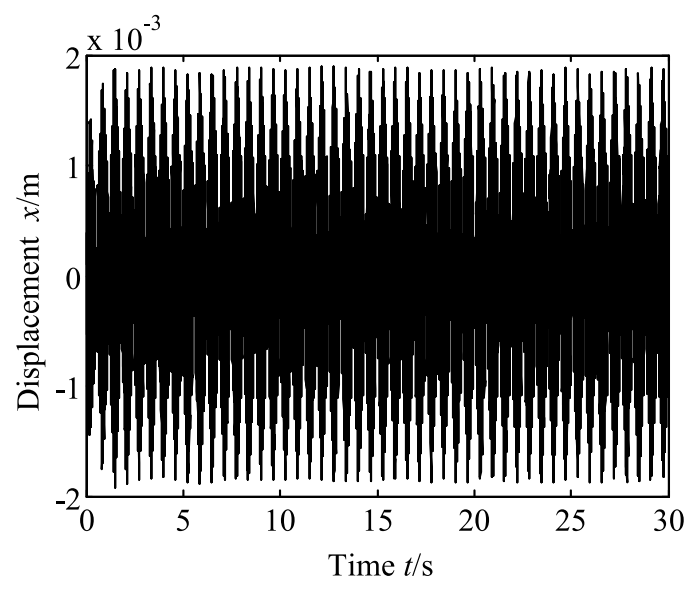

(a) Time domain signals in $x$ direction

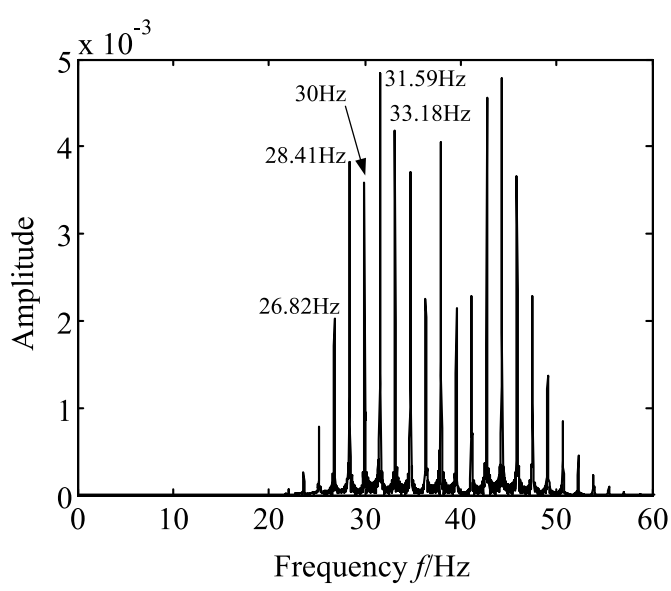

(b) Frequency spectrum

Fig. 15. Time-frequency response of rotor system with slowly varying mass $(\omega=30 \mathrm{~Hz}, \varepsilon=0.1)$. 


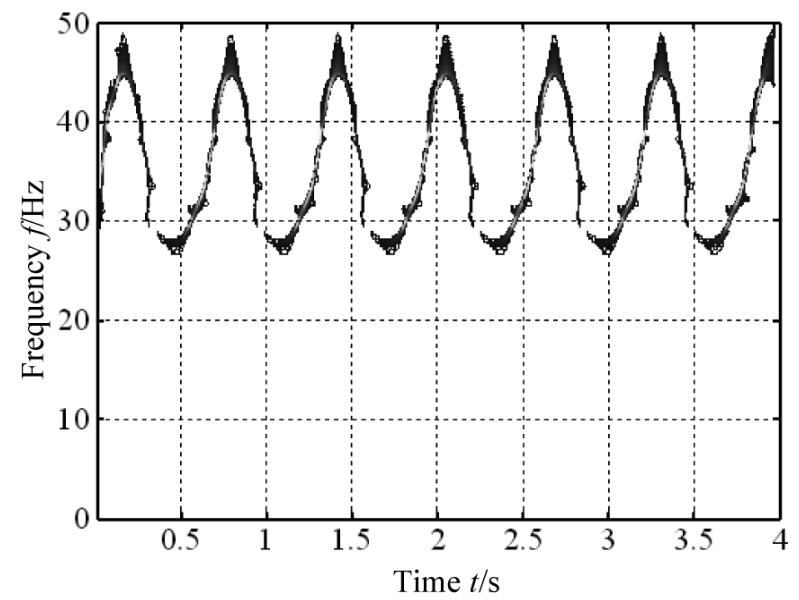

Fig. 16. The reassigned Morlet scalograms of rotor system with slowly varying mass $(\omega=30 \mathrm{~Hz}, \varepsilon=0.1)$.

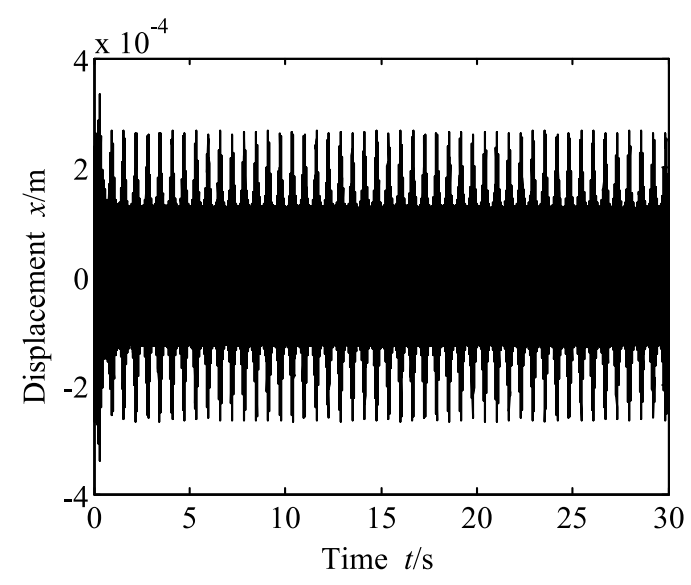

(a) Time domain signals in $x$ direction

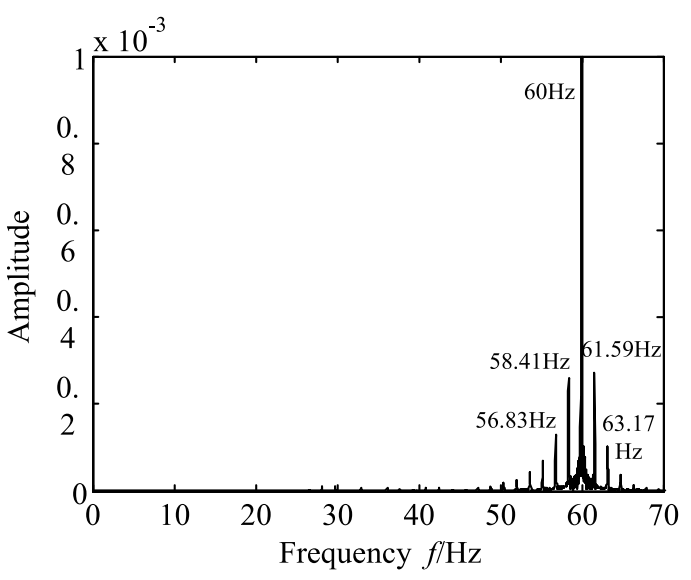

(b) Frequency spectrum

Fig. 17. Time-frequency response of rotor system with slowly varying mass $(\omega=60 \mathrm{~Hz}, \varepsilon=0.1)$.

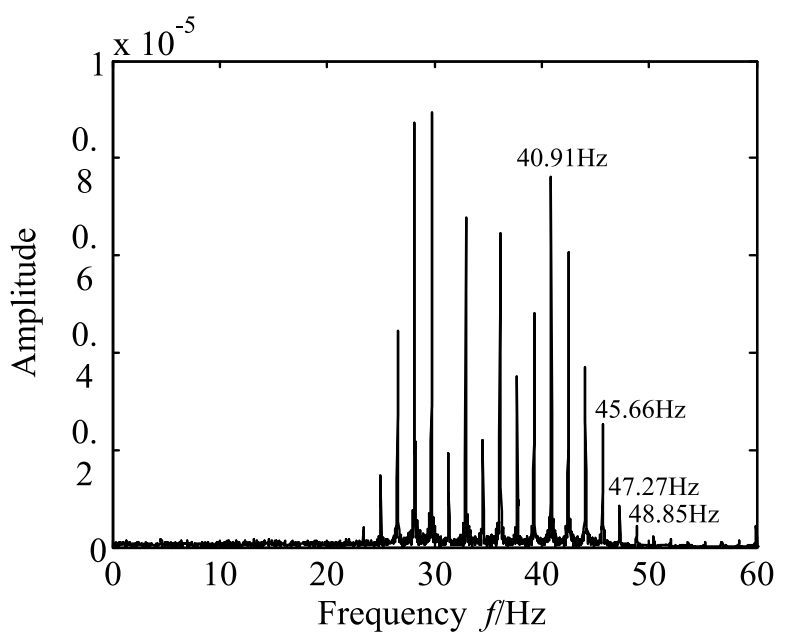

Fig. 18. Frequency spectrum of rotor system with slowly varying mass after low-pass filtering $(\omega=60 \mathrm{~Hz}, \varepsilon=0.1)$. 


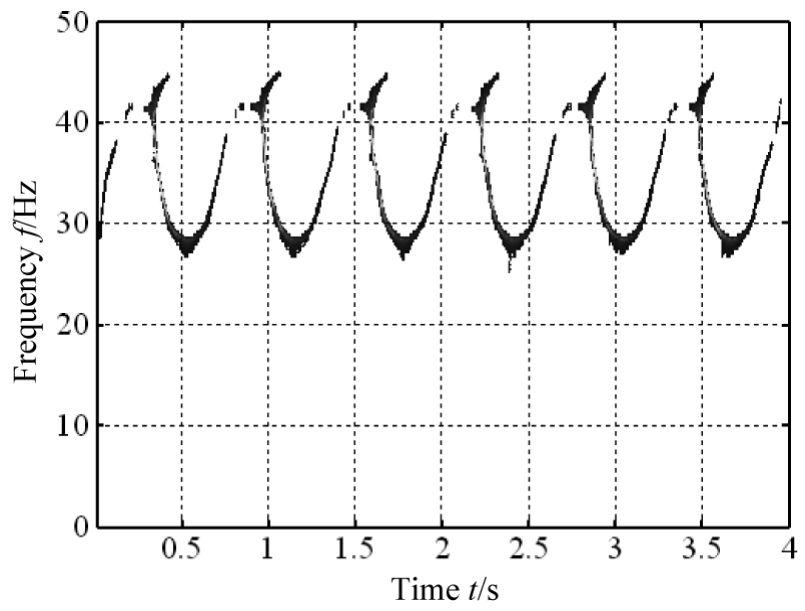

Fig. 19. The reassigned Morlet scalograms of rotor system with slowly varying mass after low-pass filtering $(\omega=60 \mathrm{~Hz}, \varepsilon=0.1)$.

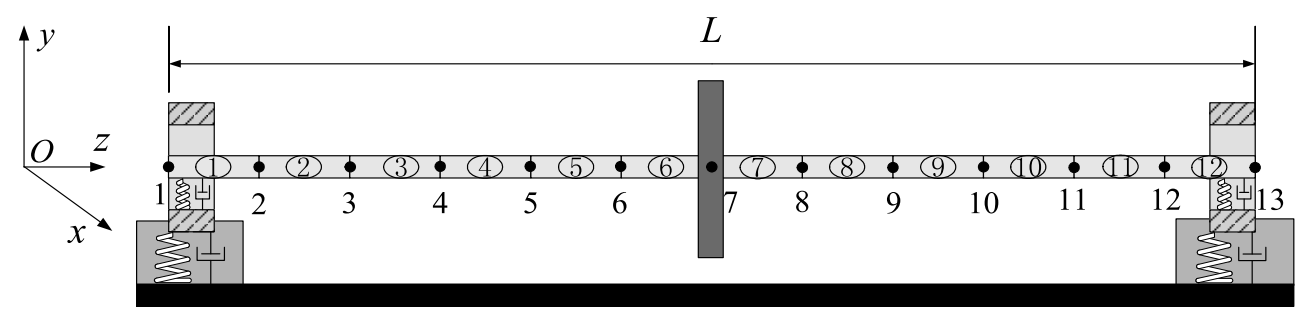

Fig. 20. Finite element model of rotor system with slowly varying mass.

According to the above time-frequency analysis of rotor system with slowly varying mass, the following conclusion can be obtained. With the given slowly varying mass model $m(\tau)=m_{0}\left(1+\lambda \cos \omega_{m} \tau\right)$, the system frequency fluctuates with a period of $T=2 \pi / \omega_{m} \varepsilon$ and an incremental of $\Delta f=\omega_{m} \varepsilon / 2 \pi$, which is related with slowly varying frequency $\omega_{m}$ and slowly varying coefficient $\varepsilon$. For the cases discussed above, the fluctuating period is $T=2 \pi /(100 \times 0.01)=2 \pi$ or $T=2 \pi /(100 \times 0.1)=\pi / 5$, which can be observed in the above time-domain figures and reassigned Morlet scalograms.

\section{Time frequency features of rotor system with slowly varying mass based on FEM}

In the previous chapter, by using asymptotic method, the analytic solutions of rotor system with single slowly varying mass are derived and simulated. For the simplicity of rotor system and approximation of analytic solution, the results are hard to avoid some errors. Furthermore, to deduce the analytic solution of a complicate rotor system is not an easy task. So, to investigate the dynamics of rotor system with multiple slowly varying mass, the finite element model of it is adopted. Since the establishment of a finite element model of rotor system has been reported in many papers [14], here the unnecessary details will not be given for the space.

\subsection{Case of single slowly varying mass}

Here, in order to compare the time frequency features of rotor system obtained with the analytic solution and numerical method, the finite element model of rotor system with single slowly varying mass, shown in Fig. 20, is studied first.

Consider the same slowly varying mass model $m(\tau)=m_{0}\left(1+\lambda \cos \omega_{m} \tau\right)$, under given model parameters $m_{0}=$ $1 \mathrm{~kg}, \omega_{m}=100 \mathrm{rad} / \mathrm{s}, \varepsilon=0.1, \lambda=0.5, R=10 \mathrm{~mm}, L=480 \mathrm{~mm}, e=0.0001 \mathrm{~m}$ and $E=2.06 \mathrm{e} 11 \mathrm{~N} / \mathrm{m}^{2}$, the natural frequency change between $26.4 \mathrm{~Hz}$ and $41.5 \mathrm{~Hz}$. 


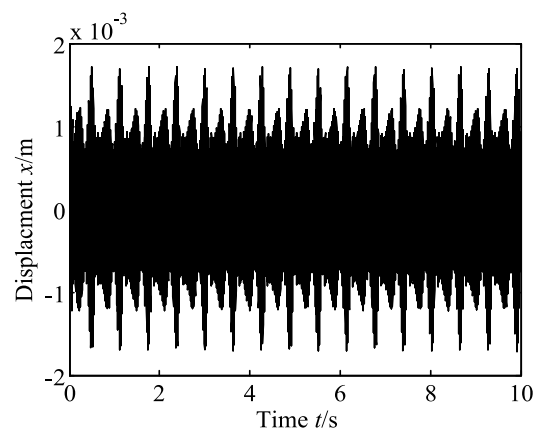

(a) Time domain signals in $x$ direction

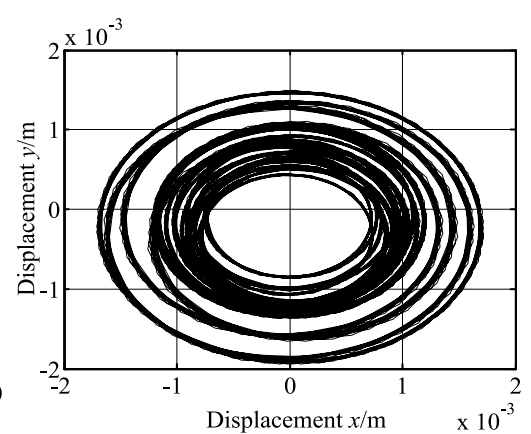

(b) Rotor obit

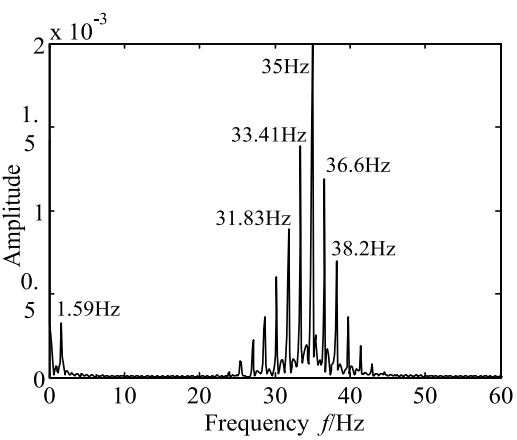

(c) Frequency spectrum

Fig. 21. Time-frequency response of rotor system with single slowly varying mass based on FEM $(\omega=35 \mathrm{~Hz}, \varepsilon=0.1)$.

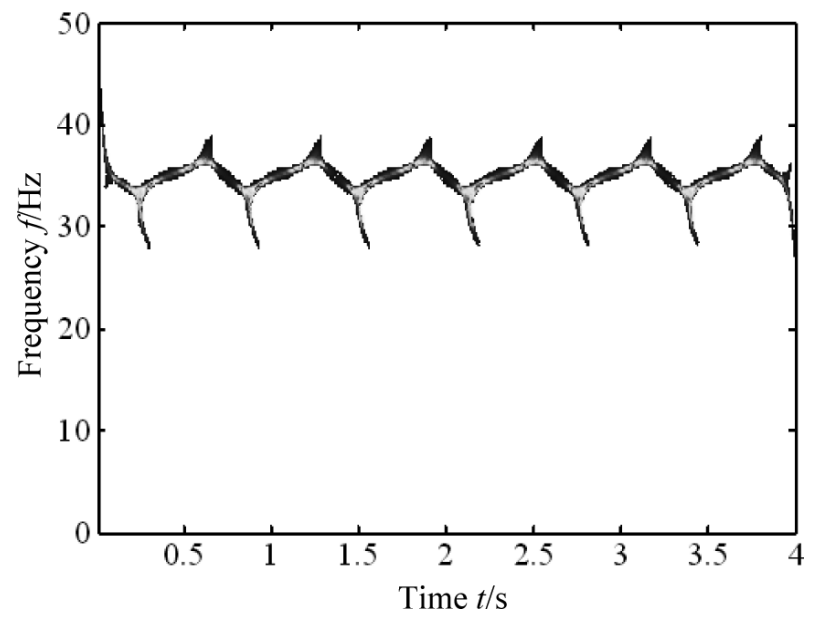

Fig. 22. The reassigned Morlet scalograms of rotor system with single slowly varying mass based on FEM $(\omega=35 \mathrm{~Hz}, \varepsilon=0.1)$.

In the modeling of mass matrix of rotor system, by replacing the constant disc mass with time-varying mass, the assembled mass matrix of rotor system become time-varying matrix. Similarly, the unbalance force and gravity vector in the dynamic equation of rotor system should be changed too. Figures 21 and 22 give us the time-frequency response and reassigned Morlet scalorgrams of rotor system with single slowly varying mss under rotating speed with $35 \mathrm{~Hz}$.

By investigating Figs 15 and 21, we find there is no big difference between the results of analytic solution and those of FEM. But what should be point out is the low frequency component in frequency spectrum of Fig. 21, i.e. $1.59 \mathrm{~Hz}$, which is the slowly varying frequency in the time $t$ scale, it can be given as $\Delta f=\omega_{m} \varepsilon / 2 \pi$. Through the comparison, we can find that the responses based on the FEM can show the detail frequency information better, which resulted from the unlimited of solution order of dynamic equations.

\subsection{Case of dual slowly varying mass}

Basing on the previous investigation of single slowly varying mass rotor system, we can study the dynamics of rotor system with dual slowly varying mass, as shown in Fig. 23, which can represent the dynamic characteristics of rotor system with multiple slowly varying mass.

Assume the models two slowly varying mass are same as $m(\tau)=m_{0}\left(1+\lambda \cos \omega_{m} \tau\right)$. To investigate the influence of different slowly varying parameters on the dynamics of rotor system, assume the slowly varying frequencies of two discs are $\omega_{m 1}=100 \mathrm{rad} / \mathrm{s}$ and $\omega_{m 2}=200 \mathrm{rad} / \mathrm{s}$ respectively, and the others are same with the ones of previous chapter. The simulation of rotor system with the above parameters are given from Figs 24 to 25 . 


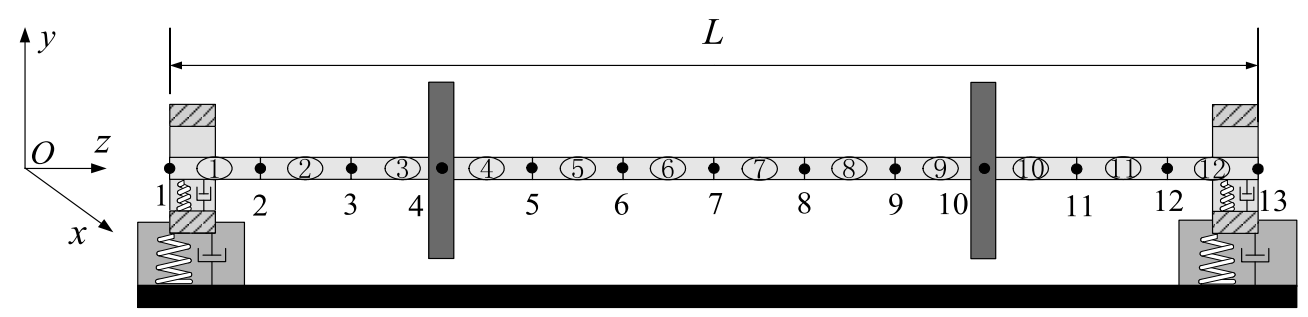

Fig. 23. Finite element model of rotor system with dual slowly varying mass.

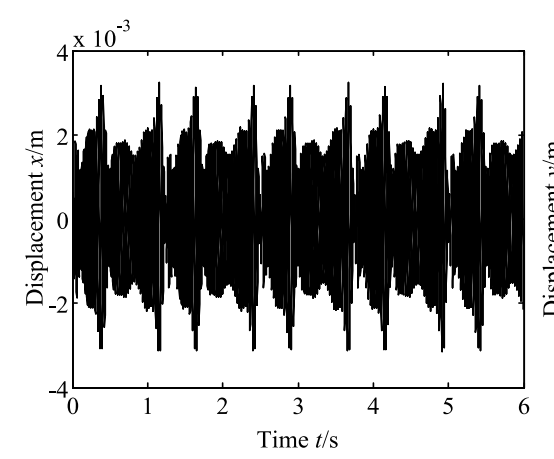

(a)Time domain signals in $x$ direction

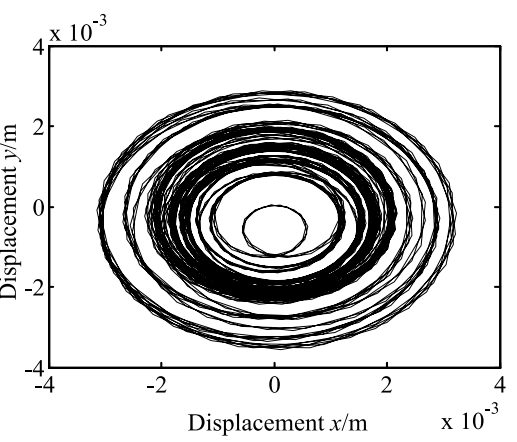

(b) Rotor obit

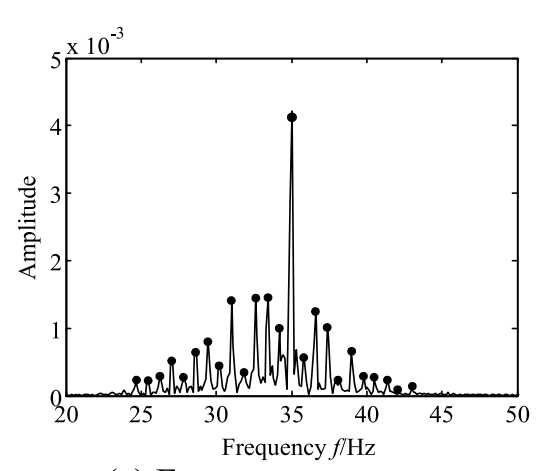

(c) Frequency spectrum

Fig. 24. Time-frequency response of rotor system with dual slowly varying mass $\left(\omega=35 \mathrm{~Hz}, \omega_{m 1}=100 \mathrm{rad} / \mathrm{s}, \omega_{m 2}=200 \mathrm{rad} / \mathrm{s}, \varepsilon=0.1\right)$.

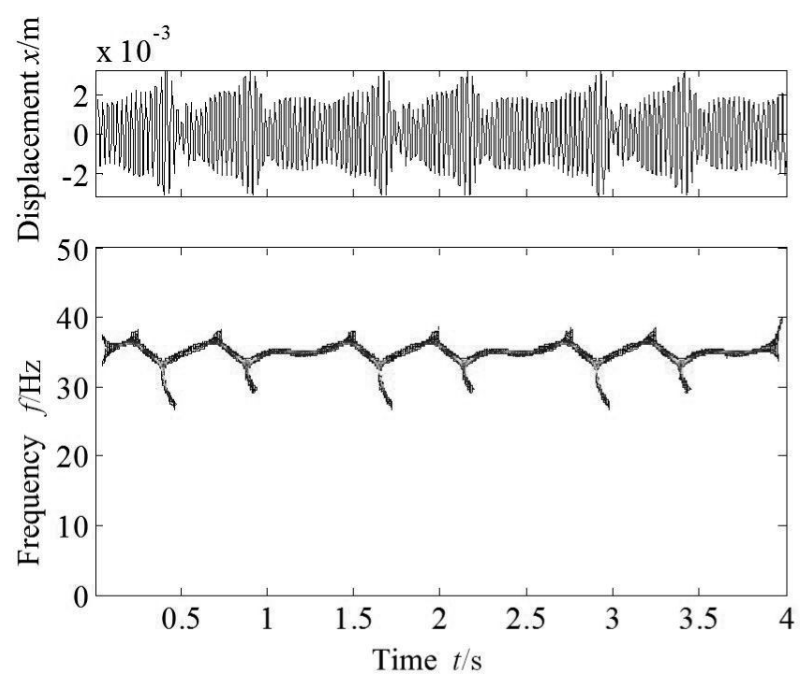

Fig. 25. The reassigned Morlet scalograms of rotor system with dual slowly varying mass $\left(\omega=35 \mathrm{~Hz}, \omega_{m 1}=100 \mathrm{rad} / \mathrm{s}, \omega_{m 2}=200 \mathrm{rad} / \mathrm{s}\right.$, $\varepsilon=0.1)$.

For the rotor system with dual slowly varying mass, the time-frequency response is more complicated than that with single slowly varying mass. In the frequency spectrum, as shown in Fig. 24(c), we find the abundant frequency components around the working frequency, which are related with slowly varying frequency and slowly varying coefficient $\varepsilon$. The frequency components in frequency spectrum, as dotted in Fig. 24(c), are listed in Table 1, where $f$ is working frequency, and $f_{1}=\omega_{m 1} \varepsilon_{1} / 2 \pi, f_{2}=\omega_{m 2} \varepsilon_{2} / 2 \pi, \omega_{m 1}=100 \mathrm{rad} / \mathrm{s}, \omega_{m 2}=200 \mathrm{rad} / \mathrm{s}, \varepsilon_{1}=\varepsilon_{2}=0.1$.

By investigating the Table 1 and Fig. 24(c), the conclusion can be made that the frequency components are distributed symmetrically around the working frequency, and they meet the following formula: 
Table 1

Components of frequency spectrum of rotor system with dual slowly varying mass $(\omega=35 \mathrm{~Hz}$, $\left.\omega_{m 1}=100 \mathrm{rad} / \mathrm{s}, \omega_{m 2}=200 \mathrm{rad} / \mathrm{s}, \varepsilon=0.1\right)$

\begin{tabular}{cccc}
\hline $\begin{array}{c}\text { Frequency } \\
\text { components }\left(f_{L}\right) \text { in } \\
\text { left side of } f(\mathrm{~Hz})\end{array}$ & $\begin{array}{c}\text { The construction } \\
\text { of frequency } f_{L}\end{array}$ & $\begin{array}{c}\text { Frequency } \\
\text { components }\left(f_{R}\right) \text { in } \\
\text { right side of } f(\mathrm{~Hz})\end{array}$ & $\begin{array}{c}\text { The construction } \\
\text { of frequency } f_{R}\end{array}$ \\
\hline 35 & $f-\left(f_{m 2}-f_{m 1}\right)$ & 35 & $f$ \\
34.18 & $f-f_{m 1}$ & 35.889 & $f+\left(f_{m 2}-f_{m 1}\right)$ \\
33.447 & $f-f_{m 2}$ & 36.621 & $f+f_{m 1}$ \\
32.593 & $f-2 f_{m 1}$ & 37.354 & $f+f_{m 2}$ \\
31.860 & $f-\left(f_{m 2}+f_{m 1}\right)$ & 38.086 & $f+2 f_{m 1}$ \\
31.006 & $f-2 f_{m 2}$ & 38.94 & $f+\left(f_{m 2}+f_{m 1}\right)$ \\
30.273 & $f-\left(f_{m 2}+2 f_{m 1}\right)$ & 39.795 & $f+2 f_{m 2}$ \\
29.419 & $f-\left(2 f_{m 2}+f_{m 1}\right)$ & 40.527 & $f+\left(2 f_{m 2}+f_{m 1}\right)$ \\
28.687 & $f-\left(f_{m 2}+3 f_{m 1}\right)$ & 42.115 & $f+\left(f_{m 2}+3 f_{m 1}\right)$ \\
27.832 & $f-2\left(f_{m 2}+f_{m 1}\right)$ & 42.969 & $f+2\left(f_{m 2}+f_{m 1}\right)$ \\
26.978 & $f-\left(f_{m 2}+4 f_{m 1}\right)$ & & \\
26.245 & $f-4 f_{m 2}$ & & \\
25.391 & \multicolumn{2}{c}{} &
\end{tabular}

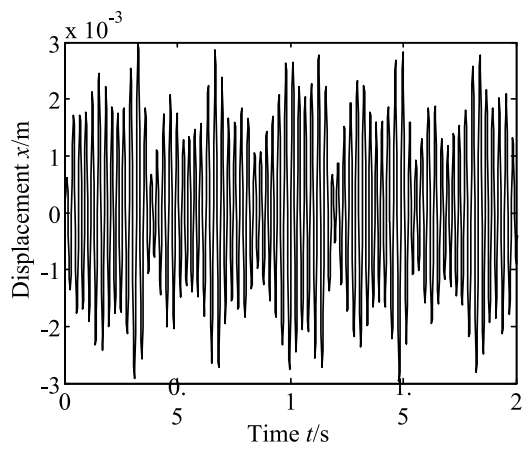

(a) Time domain signals in $x$ direction

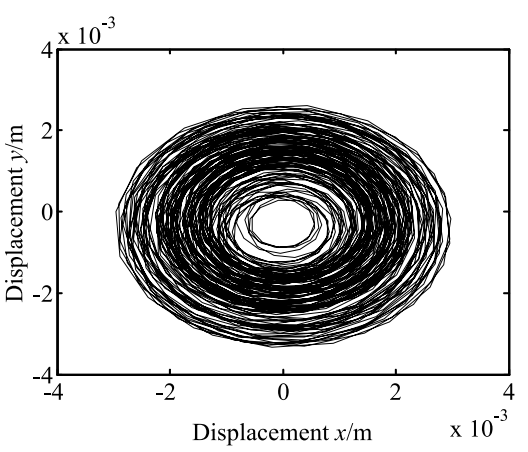

(b) Rotor obit

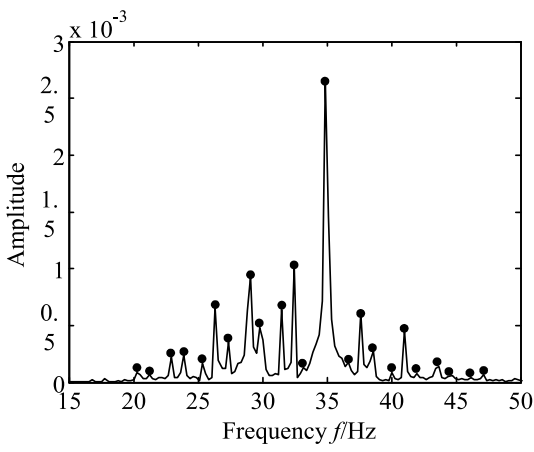

(c) Frequency spectrum

Fig. 26. Time-frequency response of rotor system with dual slowly varying mass $\left(\omega=35 \mathrm{~Hz}, \omega_{m 1}=160 \mathrm{rad} / \mathrm{s}, \omega_{m 2}=380 \mathrm{rad} / \mathrm{s}, \varepsilon=0.1\right)$.

$$
f_{i}=f \pm\left(m f_{m 1} \pm n f_{m 2}\right), i=1,2, \cdots, m=0,1,2, \cdots, n=0,1,2, \cdots
$$

Where $f$ is rotating frequency, $f_{m 1}$ and $f_{m 2}$ are slowly varying frequencies.

In order to validate the above conclusion, another example, as shown in Figs 26 and 27, is studied. The corresponding frequency components in frequency spectrum Fig. 26(c) are listed in Table 2. To illustrate the complication of frequency fluctuation well, the threshold is decreased intentionally in Fig. 27. Though more noise appears in Fig. 27, the strong nonlinearity of system is reflected.

By investigating the frequency components in Table 2, we find that they fit the Eq. (27) well. So, we can draw a another general conclusion that for the rotor system with multiple slowly varying mass, which changes at slowly varying frequencies $\omega_{m 1}, \omega_{m 2}, \omega_{m 3}, \ldots, \omega_{m j}$ and slowly vary time coefficient $\varepsilon_{1}, \varepsilon_{2}, \varepsilon_{3}, \ldots, \varepsilon_{j}$, the frequency response of the rotor system distribute symmetrically around working frequency in the frequency spectrum and meet the formula:

$$
\begin{gathered}
f_{i}=f \pm\left(n_{1} f_{m 1} \pm n_{2} f_{m 2} \pm n_{3} f_{m 3} \cdots \pm n_{j} f_{m j}\right), n_{j}=0,1,2, \cdots, j=1,2, \cdots, i=1,2, \cdots \\
\text { and } f_{i}>0,\left(n_{1} f_{1} \pm n_{2} f_{2} \pm n_{3} f_{3} \cdots \pm n_{j} f_{j}\right)>0
\end{gathered}
$$

Where $f$ is rotating frequency, $f_{m j}$ is slowly varying frequencies.

\section{Conclusions}

With the analytic method based on asymptotic method and the numerical method based on finite element method, the nonlinear dynamic response of rotor system with single slowly varying mass is studied. The analytic solution of 
Table 2

Components of frequency spectrum of rotor system with dual slowly varying mass. $(\omega=$ $\left.35 \mathrm{~Hz}, \omega_{m 1}=160 \mathrm{rad} / \mathrm{s}, \omega_{m 2}=380 \mathrm{rad} / \mathrm{s}, \varepsilon=0.1\right)$

\begin{tabular}{cccc}
\hline $\begin{array}{c}\text { Frequency } \\
\text { components }\left(f_{L}\right) \text { in } \\
\text { left side of } f(\mathrm{~Hz})\end{array}$ & $\begin{array}{c}\text { The construction } \\
\text { of frequency } f_{L}\end{array}$ & $\begin{array}{c}\text { Frequency } \\
\text { components }\left(f_{R}\right) \text { in } \\
\text { right side of } f(\mathrm{~Hz})\end{array}$ & $\begin{array}{c}\text { The construction } \\
\text { of frequency } f_{R}\end{array}$ \\
\hline 35 & $f$ & 35 & $f$ \\
33.447 & $f-\left(3 f_{m 1}-f_{m 2}\right)$ & 36.621 & $f+\left(3 f_{m 1}-f_{m 2}\right)$ \\
32.471 & $f-f_{m 1}$ & 37.598 & $f+f_{m 1}$ \\
31.494 & $f-\left(f_{m 2}-f_{m 1}\right)$ & 38.574 & $f+\left(f_{m 2}-f_{m 1}\right)$ \\
30.029 & $f-2 f_{m 1}$ & 40.039 & $f+2 f_{m 1}$ \\
29.053 & $f-f_{m 2}$ & 41.016 & $f+f_{m 2}$ \\
27.344 & $f-3 f_{m 1}$ & 42.725 & $f+3 f_{m 1}$ \\
26.367 & $f-\left(f_{m 2}+f_{m 1}\right)$ & 43.457 & $f+\left(f_{m 2}+f_{m 1}\right)$ \\
25.390 & $f-\left(2 f_{m 2}-f_{m 1}\right)$ & 44.434 & $f+\left(2 f_{m 2}-f_{m 1}\right)$ \\
24.902 & $f-4 f_{m 1}$ & & $f+\left(f_{m 2}+2 f_{m 1}\right)$ \\
23.926 & $f-\left(f_{m 2}+2 f_{m 1}\right)$ & 46.143 & $f+2 f_{m 2}$ \\
22.949 & $f-2 f_{m 2}$ & 47.119 & \\
21.484 & $f-\left(f_{m 2}+3 f_{m 1}\right)$ & & \\
20.264 & $f-\left(3 f_{m 2}-f_{m 1}\right)$ & &
\end{tabular}

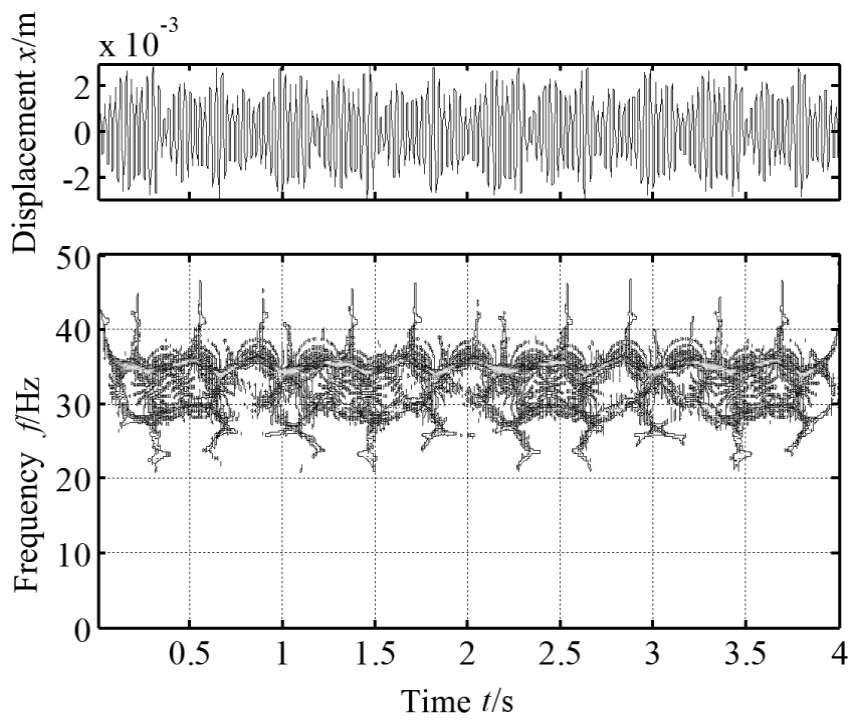

Fig. 27. The reassigned Morlet scalograms of rotor system with dual slowly varying mass $\left(\omega=35 \mathrm{~Hz}, \omega_{m 1}=160 \mathrm{rad} / \mathrm{s}, \omega_{m 2}=380 \mathrm{rad} / \mathrm{s}\right.$, $\varepsilon=0.1)$.

rotor system with slowly varying mass, based on asymptotic method, depends on the approximate extent. For the first order approximate solution is used, there exists difference between the results of the analytic method and those of FEM. The later one can reflect not only the detailed frequency information absent by using analytic method, but also the better frequency fluctuation of rotor system.

By analyzing the rotor system with dual slowly varying mass, the frequency response of system are found distributing in the area of the two side of rotating frequency in the frequency spectrum with the increasing or decreasing combination frequency, which is related with the product of slowly varying frequency $\omega_{m}$ and slowly varying time coefficient $\varepsilon$, i.e. the frequency in time t scale. At last, on the basis of common characteristics of rotor system with dual slowly varying mass, the general regulation and formula describing the frequency distribution of rotor system with multiple slowly varying mass are proposed. 


\section{Acknowledgements}

The authors gratefully acknowledge that the work was supported by the Key Project of Science and Technology Research Funds of Chinese Ministry of Education (Granted No. 108037) and the National High Technology Research and Development Program of China (863 Program) (No. 2007 AA 04Z418).

\section{References}

[1] B.C. Wen, X.H. Wu, Q. Ding and Q.K. Han, Dynamics and Experiments of Fault Rotors, Science Press, Beijing, 2004.

[2] Y.A. Mitropoliski, The Unstable Process of the Nonlinear Vibration System, Science Press, China, 1958.

[3] S.M. Shahruz and C.A. Tan, Response of linear slowly varying systems under external excitations, Journal of Sound and Vibration 131(2) (1989), 239-247.

[4] L. Cveticanin, Chaos in rotors with slowly varying mass, Journal of Sound and Vibration 185(5) (1995), 897-901.

[5] L. Cveticanin, Normal mode of vibration for continuous rotors with slow time variable mass, Mech Mach Theory 32(7) (1997), 881-891.

[6] B. Wen et al., Vibration characteristics of the nonlinear system with slow- changing parameters, Journal of Nonlinear Dynamics 5(2) (1998), 181-188, in Chinese.

[7] Y.N. Li, L.Z. and B.C. Wen, Study of dynamic characteristics of slow-changing process, Shock and Vibration 7 (2000), 113-117.

[8] J.P. Cai, X.F. Wu and Y.P. Li, Comparison of multiple scales and KBM methods for strongly nonlinear oscillators with slowly varying parameters, Mechanics Research Communications 31(5) (2004), 519-524.

[9] J.P. Cai and Y.P. Li, Strongly non-linear oscillators with slowly varying parameters, Journal of Sound and Vibration 275(1-2) (2004), $241-248$.

[10] J.P. Cai and Y.P. Li, Approximate potentials with applications to strongly nonlinear oscillators with slowly varying parameters, Shock and Vibration 10 (2003), 379-386.

[11] M.B. Ependiyev, An asymptotic analysis of the forced oscillations in systems with slowly varying parameters, Journal of Applied Mathematics and Mechanics 53(4) (1989), 429-439.

[12] D. Mohammed and A.D. Munther, On slowly time-varying systems, Automatica 27(1) (1991), 201-205.

[13] M. Shamsul Alam, Unified Krylov-Bogoliubov-Mitropolskii method for solving nth order non-linear systems with slowly varying coefficients, Journal of Sound and Vibration 265(5) (2003), 987-1002.

[14] H.D. Nelson and J.M. Mcvaugh, The dynamics of rotor-bearing systems using finite elements, Journal of Engineering for Industry 5 (1976), 593-600. 

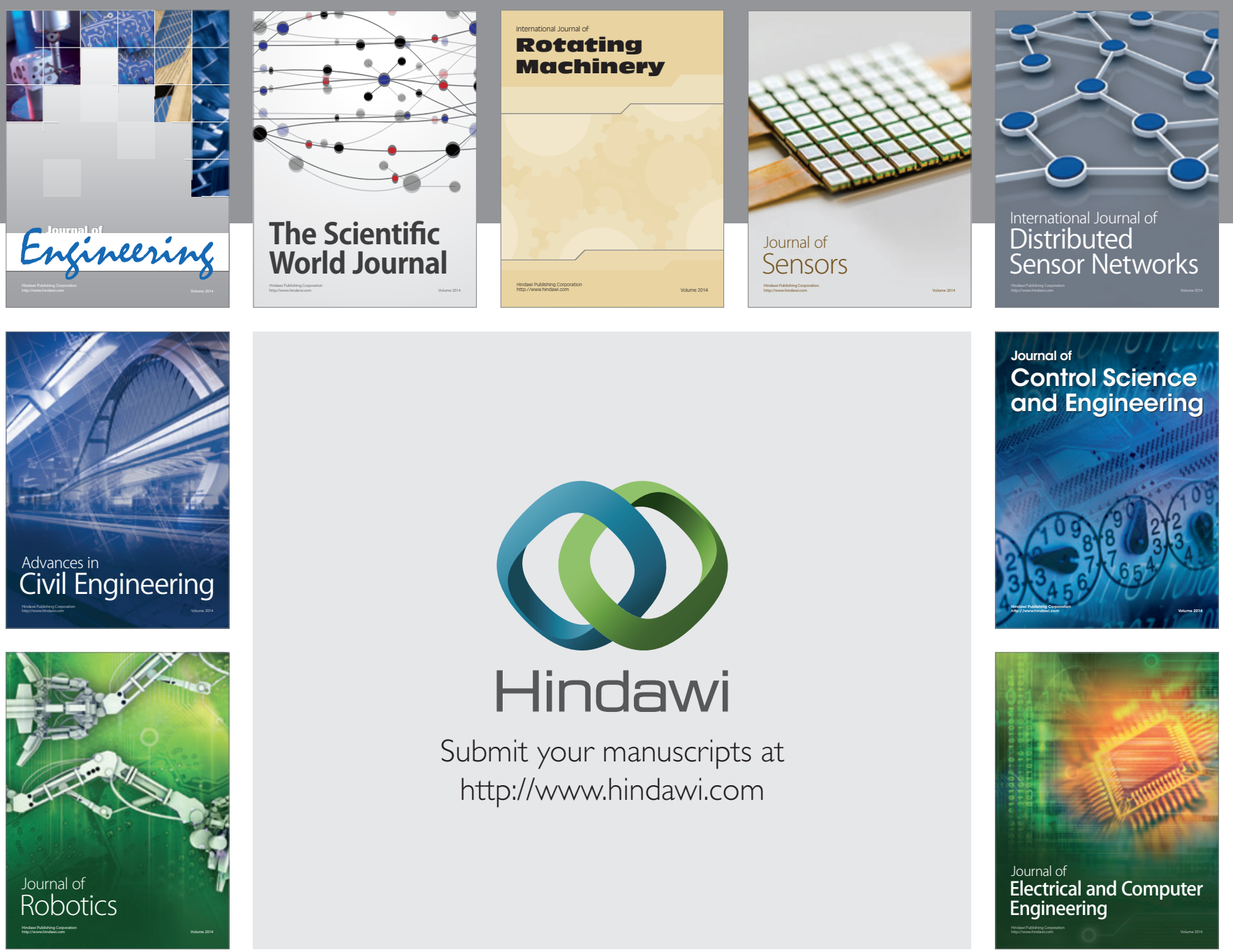

Submit your manuscripts at

http://www.hindawi.com
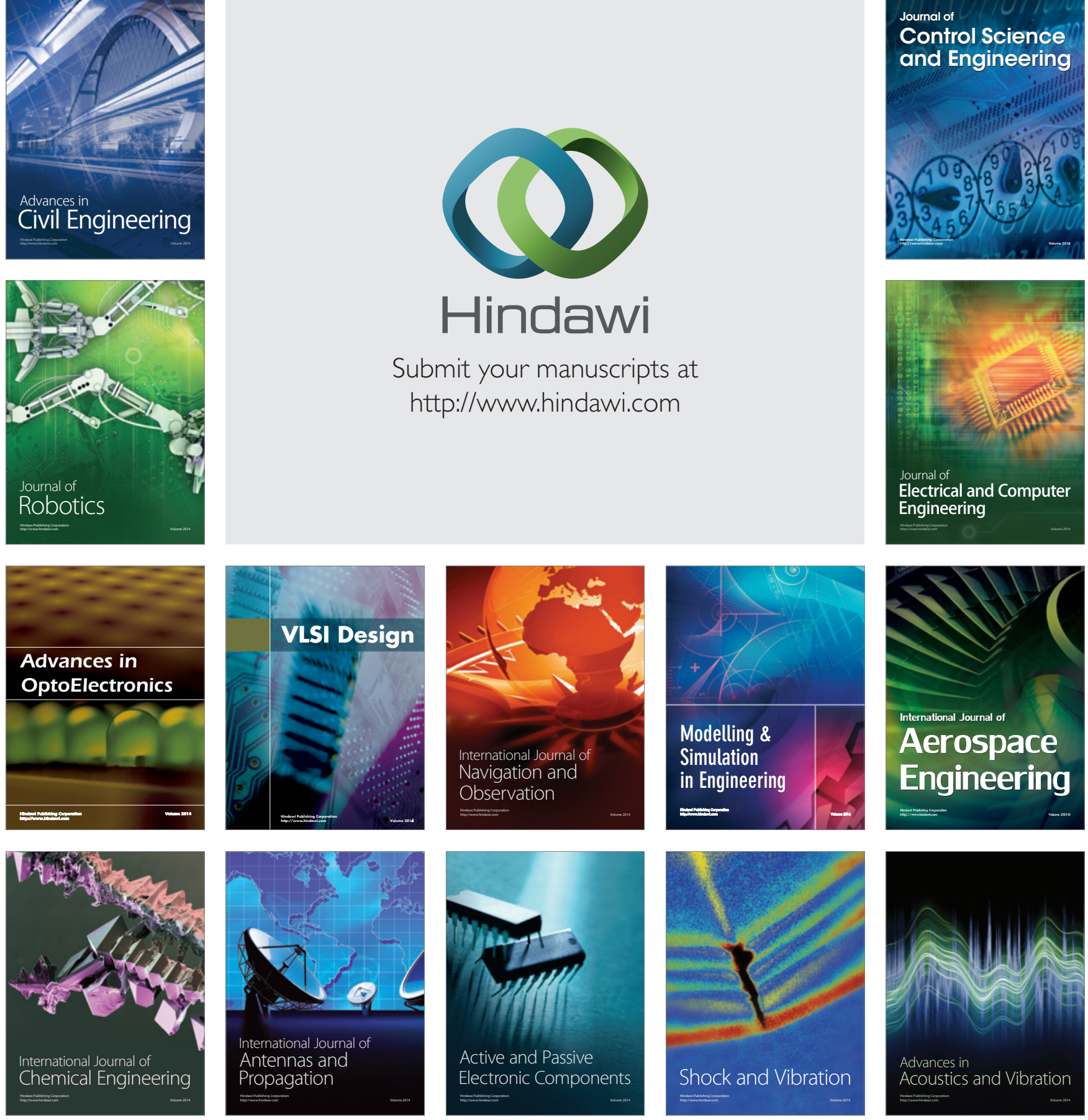